\title{
Análisis de la relación entre nivel de dependencia del paciente en hemodiálisis y sobrecarga del cuidador principal
}

\author{
Azahara Contreras Gallego, Pablo Jesús López Soto, Rodolfo Crespo Montero
}

\section{Hospital Universitario Reina Sofía. Córdoba}

\section{Introducción:}

Los pacientes en hemodiálisis (HD) presentan alteraciones asociadas no solo con la edad sino también con comorbilidad añadida. Debido a estas características, el cuidado del paciente en HD es cada vez más complejo, ya que presentan mayor discapacidad física, menor autonomía y capacidad de autocuidado, recayendo gran parte de sus cuidados en un familiar directo o cuidador principal, que acaban siendo partícipes de los cuidados al paciente, y en ocasiones, sufriendo lo que se conoce como la "sobrecarga del cuidador". El objetivo de este estudio fue analizar la relación entre el grado de dependencia de los pacientes en HD y la sobrecarga de su cuidador.

\section{Pacientes y métodos:}

Se realizó un estudio observacional analítico. Se estudiaron 36 pacientes (43\% varones, 57\% mujeres) del programa de HD del servicio de Nefrología de una hospital terciario. Se incluyó en la muestra todos los pacientes que tuvieran algún grado de dependencia (Barthel $<100$ ) y sus respectivos cuidadores, siempre que no fueran remunerados. Se estudió: edad y género del cuidador y del paciente, tipo de transporte sanitario, tiempo en HD, nivel de sobrecarga mediante el cuestionario de Zarit, nivel de dependencia mediante el test Delta y el índice de Barthel.

\section{Resultados:}

En los pacientes, al realizar el test Delta observamos como la dependencia media es de $11.05 \pm 7.79$, es decir un tercio de los pacientes estaban en la categoría de asistidos moderados, la dependencia física se situó en $5.86 \pm 3.03$ (déficit moderado) y la dependencia psíquica en $3.11 \pm 2.95$ (ausencia de déficit). Con respecto al índice de Barthel, la media de los pacientes fue de $58.30 \pm 29.17$ (dependencia severa). Cuando agrupamos a los mismos por categorías dentro de este índice, encontramos: 19 (53\% pacientes con dependencia moderada, $13(36 \%)$ con dependencia severa y $411 \%$ ) con dependencia total. Los cuidadores principales fueron un $64 \%$ mujeres y $36 \%$ varones, y con una edad media de $64.44 \pm 16$ años. La sobrecarga media de los cuidadores principales fue $45.92 \pm 16$, por tanto a nivel global, no existe sobrecarga, pero cuando se distribuyeron por las categorías del Zarit 5 (14\%) presentaron sobrecarga moderada y $8(33 \%)$ sobrecarga intensa. Cuando se relacionó la puntuación obtenida por los pacientes en el índice de Barthel con la sobrecarga del cuidador, se encontró una correlación inversa entre ambos, siendo estadísticamente significativa $(r=-0.3861, p=0.02)$; es decir a mayor puntuación de Barthel (más independencia del paciente) menor puntuación de Zarit del cuidador (menos saturación del cuidador). Por tanto, no se cumple la hipótesis que nos planteamos al realizar este estudio.

\section{Conclusiones:}

El nivel de dependencia de nuestros pacientes es similar al encontrado en la mayoría de las series estudiadas, pues casi la mitad tienen dependencia total o severa. Sin embargo, la sobrecarga del cuidador no es tan alta, pues solo presentan signos de sobrecarga menos de la mitad de los cuidadores. Esta sobrecarga no tiene relación con el nivel de dependencia de los pacientes. 